
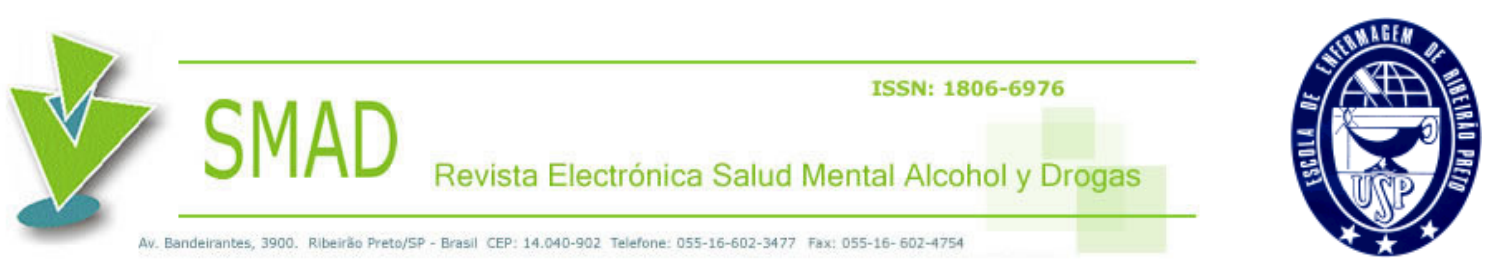

\title{
O USO DA MACONHA ASSOCIADO AO DÉFICIT DE HABILIDADES SOCIAIS EM ADOLESCENTES
}

\section{Márcia Fortes Wagner ${ }^{1}$; Jaqueline Garcia da Silva ${ }^{2}$; Luciana Bohrer Zanettelo ${ }^{3}$; Margareth da Silva Oliveira ${ }^{4}$}

A proposta deste trabalho foi a realização de estudo comparativo, a partir da descrição de dados de avaliação das habilidades sociais de uma amostra de adolescentes. A amostra foi composta por 30 sujeitos, sendo 15 adolescentes usuários de maconha e 15 não usuários da mesma substância. Os achados sugerem que a área mais deficitária na população de usuários de maconha relaciona-se ao autocontrole da agressividade a situações aversivas, e inabilidade para lidar com sentimentos e reações gerados nessas situações. Um dos fatores que contribui para o uso de substâncias é o fato de familiares também usarem drogas.

Descritores: Relações Interpessoais; Adolescente; Cannabis.

\section{The Association between Marijuana USE and Social Skills Deficit IN AdOLESCENTS}

The purpose of this article is to present a comparative study based on the description of the data from a social skills evaluation in a sample of adolescents. The sample consisted of 30 adolescent subjects, 15 were marijuana users and 15 were non-users. The findings suggest that the aspect with the greatest deficit in the population of marijuana users was their selfcontrol of aggressiveness in aversive situations, and the inability to deal with feelings and reactions that arise in those situations. One factor that contributes with the substance use is having family members that also use drugs.

Descriptors: Interpersonal Relations; Adolescent; Cannabis.

\footnotetext{
1-Psicóloga, Doutoranda em Psicologia pela Pontifícia Universidade Católica do Rio Grande do Sul. Bolsista da Coordenação de Aperfeiçoamento de Pessoal de Nível Superior (CAPES) no Grupo de Pesquisa "Avaliação e Atendimento em Psicoterapia Cognitiva". Professor Adjunto da Escola de Psicologia da Faculdade Meridional de Passo Fundio, RS, Brasil.

2-Psicóloga, Mestranda em Psicologia Clínica pela Pontifícia Universidade Católica do Rio Grande do Sul. Bolsista CNPq no Grupo de Pesquisa "Avaliação e Atendimento em Psicoterapia Cognitiva”.

3-Psicóloga pela Pontifícia Universidade Católica do Rio Grande do Sul.

4-Psicóloga, Doutora em Ciências pela Universidade Federal de São Paulo. Professor Adjunto da Faculdade de Psicologia da Pontifícia Universidade Católica do Rio Grande do Sul. Coordenadora do Grupo de Pesquisa "Avaliação e Atendimento em Psicoterapia Cognitiva", da Pontifícia Universidade Católica do Rio Grande do Sul. E-mail: mwagner@via-rs.net.

Endereço para Correspondência: Pontifícia Universidade Católica do Rio Grande do Sul, Programa de Pós-Graduação em Psicologia. Avenida Ipiranga, 6681 - Prédio 11 - sala 927, Partenon, 90169-900, Porto Alegre, Rio Grande do Sul, Brasil.

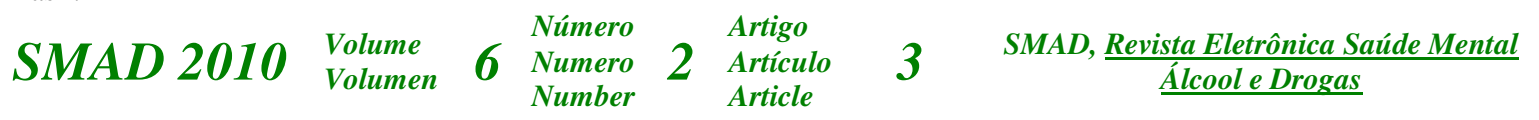




\section{EL USO DE LA MARIHUANA ASOCIADO AL DÉFICIT DE HABILIDADES SOCIALES EN ADOLESCENTES}

El presente trabajo propone la realización de un estudio comparativo, a partir de la descripción de datos de una evaluación de las habilidades sociales de una muestra de adolescentes. La muestra se compuso de 30 sujetos, 15 de ellos usuarios de marihuana y 15 no usuarios de dicha substancia. Los resultados sugieren que el área más deficitaria en la población de usuarios de marihuana se relaciona con el autocontrol de la agresividad ante situaciones adversas, e incapacidad para afrontar sentimientos y reacciones generados en esas situaciones. Uno de los factores que coadyuvan para el uso de substancias es el hecho de que otros familiares también sean usuarios de drogas.

Descriptores: Relaciones Interpersonales; Adolescente; Cannabis.

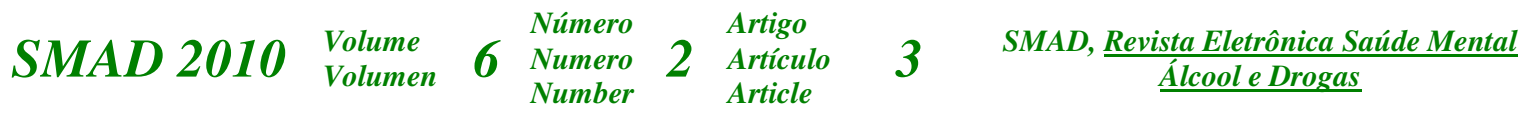



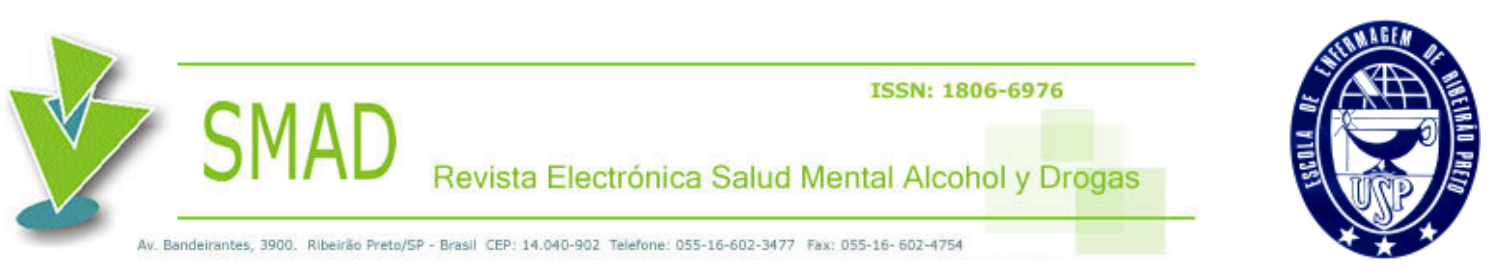

\section{Introdução}

O problema do abuso e dependência de substâncias psicoativas pode ser vivenciado com frequência na adolescência, etapa do desenvolvimento na qual surgem inúmeras transformações nas capacidades e habilidades pessoais do indivíduo. Esse é momento propício ao surgimento do consumo de drogas, pois, como o indivíduo ainda não desenvolveu de forma adequada algumas habilidades, pode ser estimulado ao uso de cigarros, álcool, maconha até ao consumo de múltiplas drogas ilícitas ${ }^{(1-2)}$.

De acordo com o II Levantamento Domiciliar sobre o Uso de Drogas Psicotrópicas no Brasil ${ }^{(3)}$, em estudo desenvolvido em 2005, com 7.939 sujeitos, nas 108 maiores cidades do país, a maconha é a droga mais facilmente encontrada e mais usada no Brasil, sendo três vezes maior o uso na vida no sexo masculino do que no sexo feminino, em todas as faixas etárias. Das Regiões brasileiras, as que mais apresentaram consumo foram a Região Sudeste $(10,3 \%)$ e a Sul $(9,7 \%)$. O DSM-IV-TR corrobora esses dados, apresentando a maconha, também, como mais frequentemente utilizada por homens, tal como a maioria das outras drogas ilícitas ${ }^{(4)}$.

Autores destacam que muitas pessoas experimentam substâncias que podem provocar dependência, mas a maioria não se torna dependente, o que revela que existem diferenças individuais referentes à vulnerabilidade à dependência, devido a fatores ambientais e genéticos. Os autores explicam que entre os fatores que predispõem à dependência de substâncias estão: a disponibilidade de drogas, o grupo de amigos,

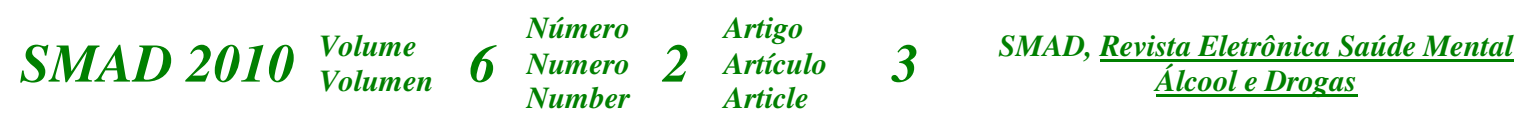



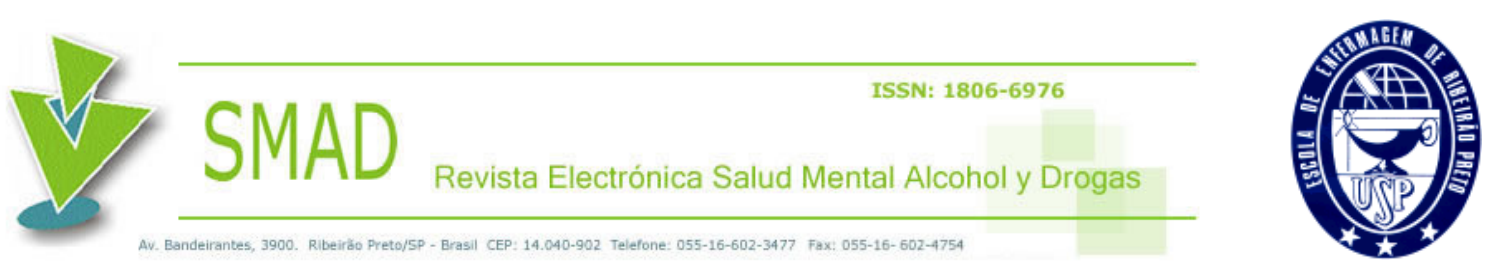

problemas familiares, déficits escolares, apoio social, resistência à pressão social e capacidade de resolução de problemas ${ }^{(5)}$.

Pesquisadores realizaram estimativa de que mais de 6 milhões de crianças vivem com pelo menos um pai que é abusador, ou dependente, de substância e conclui que crianças expostas ao abuso de drogas e álcool estão em maior risco para experimentar dificuldades acadêmicas e de comportamento. Além disso, destaca que indivíduos com problemas emocionais e comportamentais, que já estão em risco de fracasso escolar, também estão em risco aumentado para abuso de substâncias ${ }^{(6)}$.

Nesse contexto, é necessário salientar o papel das habilidades sociais como fator de proteção ao consumo de substâncias. Habilidade social ou comportamento hábil socialmente pode ser compreendido enquanto comportamentos do indivíduo que podem expressar seus sentimentos, atitudes, desejos, opiniões ou direitos de modo adequado à situação, sempre respeitando os demais, sendo que, usualmente, resolvem os problemas imediatos e minimizam a probabilidade de nova ocorrência ${ }^{(7)}$. Outros autores corroboram essa definição de habilidades sociais como um conjunto de desempenhos que o indivíduo apresenta em situações interpessoais ${ }^{(8)}$.

Alguns autores conceituam habilidades sociais como comportamentos desejáveis que propiciam a interação com o outro, dentre os quais estão a verbalização, a expressão facial, a postura, o contato visual, gestos, aparência física, entre outras. Dessa forma, quando uma pessoa se comporta de forma assertiva, facilita a solução de problemas interpessoais, aumenta a autoeficácia e a autoestima, melhora a qualidade de

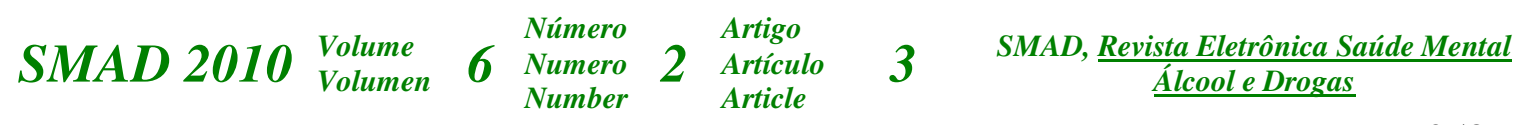



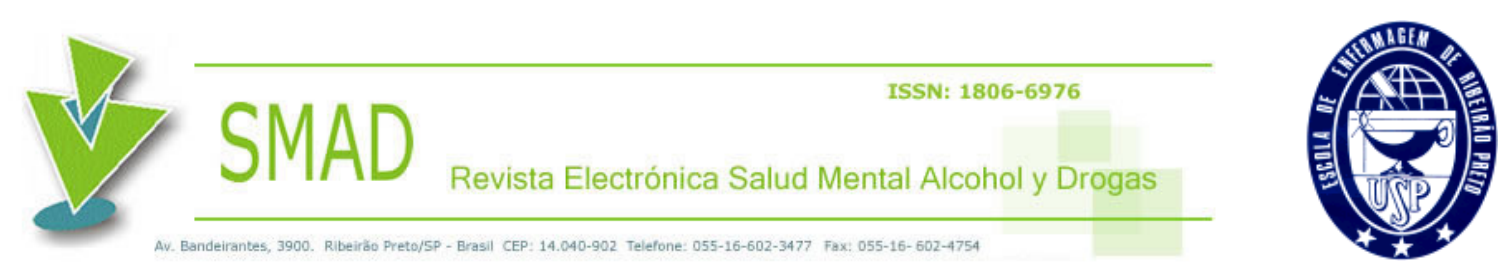

relacionamentos e promove tranquilidade ${ }^{(9-10)}$.

A literatura define assertividade como a habilidade para expressar pensamentos e sentimentos sem prejudicar os próprios direitos e preservar o direito dos outros. Pode ser considerada o oposto da agressividade, pois a pessoa assertiva expressa o que sente de forma segura e tranquila, sem precisar agredir ${ }^{(11)}$. O aprendizado de novas habilidades interpessoais capacita os indivíduos com dificuldades para serem assertivos e defenderem seus direitos de forma mais efetiva, quando houver a pressão de outras pessoas, inclusive no consumo de substâncias psicoativas ${ }^{(12)}$.

No que se refere à competência social, alguns autores ${ }^{(13)}$ enfocam a flexibilidade comportamental, os fatores culturais, relacionados a questões socioeconômicas, grupos sociais, sexo, idade etc. Alguns achados de pesquisas forneceram suporte para a afirmação de que há déficits em habilidades sociais nos adolescentes, mesmo que tais déficits de competências sociais sejam inconsistentes nos adultos ${ }^{(14)}$.

Foi realizado estudo com 314 sujeitos, entre 18 e 30 anos, usuários de drogas, para verificar consumo, estratégias de enfrentamento e habilidades sociais. As conclusões apontam para a existência de déficits relacionados a estratégias de enfrentamento e nas habilidades de relacionamento com o sexo oposto, os quais influenciam o consumo de substâncias $^{(15)}$.

Outro aspecto importante diz respeito às relações entre os construtos de influências e competências sociais no reforço à prevenção de cigarro, álcool e maconha. Tal modelo de interação forneceu evidências de que competências de recusa são úteis para estudantes que

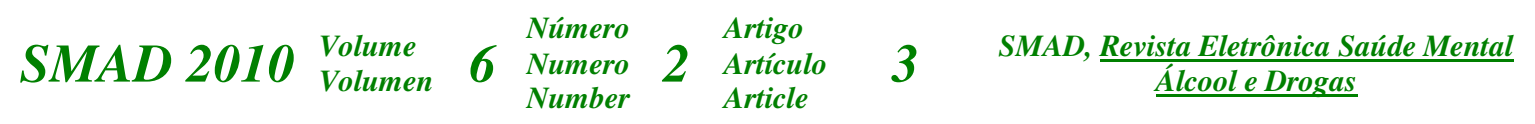



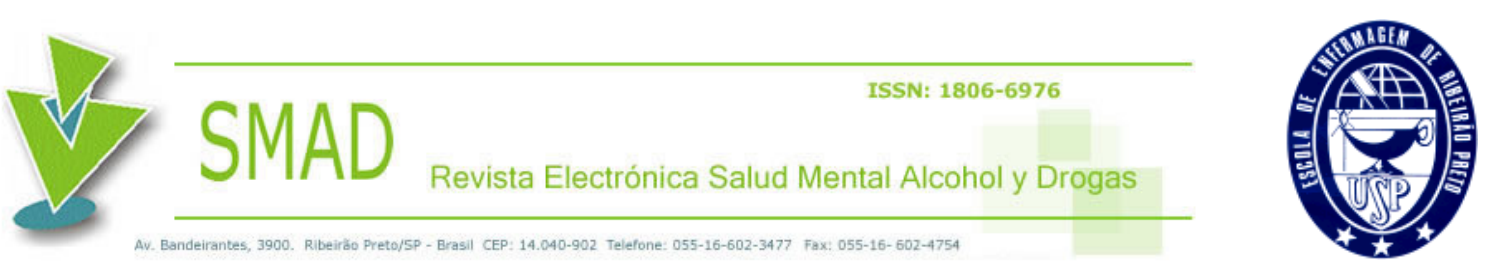

já possuem atitudes e crenças negativas sobre a substância, na tomada de decisão de não usar drogas, mas pode ser útil somente se as atitudes e percepções de danos também forem $\operatorname{abordadas}^{(16)}$.

Algumas medidas que diferenciam consequências positivas e negativas e os pesos atribuídos pelos estudantes a cada substância podem ser mais acertadamente preditivas da intenção para o uso. O álcool é o melhor exemplo, porque os jovens estão expostos e podem ter experimentado em maior grau as consequências positivas do uso, embora enfrentando as consequências negativas em menor grau. Autores corroboram esses achados e referem que bebedores mais severos apresentam mais expectativas positivas, confirmando a importância de avaliar expectativas e crenças acerca dos efeitos das substâncias para o desenvolvimento da prevenção de recaída, em programas de prevenção e reabilitação ${ }^{(17)}$.

Pesquisadores investigaram a associação entre comportamentos de uso de substâncias e seus determinantes cognitivo-sociais. Os achados sugerem que melhorar as competências gerais, como habilidades de recusa, e as cognições, como as avaliações sobre as drogas, em diferentes contextos de tipo de substância, local e prazo, pode ser caminho eficaz para a prevenção ${ }^{(18)}$.

O presente artigo objetivou descrever dados sobre a avaliação das habilidades sociais de uma amostra de adolescentes usuários de maconha, comparados com adolescentes não usuários da mesma substância.

SMAD $2010 \begin{aligned} & \begin{array}{l}\text { Volume } \\ \text { Volumen }\end{array} \\ & \text { Solumero }\end{aligned} \quad \begin{aligned} & \begin{array}{l}\text { Número } \\ \text { Number } \\ \text { Number }\end{array} \\ & 2\end{aligned} \begin{aligned} & \begin{array}{l}\text { Artigo } \\ \text { Artículo } \\ \text { Article }\end{array} \\ & \text { SMAD, Revista Eletrônica Saúde Mental }\end{aligned}$ 


\section{Material e Métodos}

O artigo é um estudo quantitativo, observacional, que compara habilidades sociais em dois grupos de adolescentes de uma amostra composta por 30 participantes: 15 pertencentes ao grupo de usuários de maconha de instituição de atendimento a dependentes químicos e 15 do grupo de não usuários, de instituição pública de ensino do Estado do Rio Grande do Sul.

Foram utilizados os seguintes instrumentos: entrevista estruturada do Manual Diagnóstico e Estatístico de Transtornos Mentais (DSM-IV-TR) para diagnóstico de abuso e dependência de substância, Inventário de Habilidades Sociais - IHS ${ }^{(19)}$, Screening Cognitivo das Escalas Weschler de Inteligência ${ }^{(20)}$ e Inventários de Ansiedade e Depressão de Beck ${ }^{(21)}$. O IHS ${ }^{(19)}$ é instrumento desenvolvido no Brasil, destinado a aferir o repertório de habilidades sociais em uma amostra de situações cotidianas, permitindo identificar déficits e recursos existentes. Possui 38 itens de autorrelato e apresenta estrutura de cinco fatores, a seguir. Fator 1 - enfrentamento com risco, relacionado a situações de afirmação, defesa de direitos e de autoestima, Fator 2 - autoafirmação na expressão de afeto positivo, que retrata expressão de afeto positivo e de afirmação da autoestima. Fator 3 - conversação e desenvoltura social, relativo a situações de "traquejo social" na conversação. Fator 4 autoexposição a desconhecidos ou a situações novas, incluindo a abordagem a pessoas

$$
\text { SMAD } 2010 \begin{aligned}
& \begin{array}{l}
\text { Volume } \\
\text { Volumen }
\end{array} \\
& \text { Solumero }
\end{aligned} \quad \begin{aligned}
& \begin{array}{l}
\text { Número } \\
\text { Number } \\
\text { Number }
\end{array} \\
& 2
\end{aligned} \begin{aligned}
& \begin{array}{l}
\text { Artigo } \\
\text { Artículo } \\
\text { Article }
\end{array} \\
& \text { SMAD, Revista Eletrônica Saúde Mental }
\end{aligned}
$$



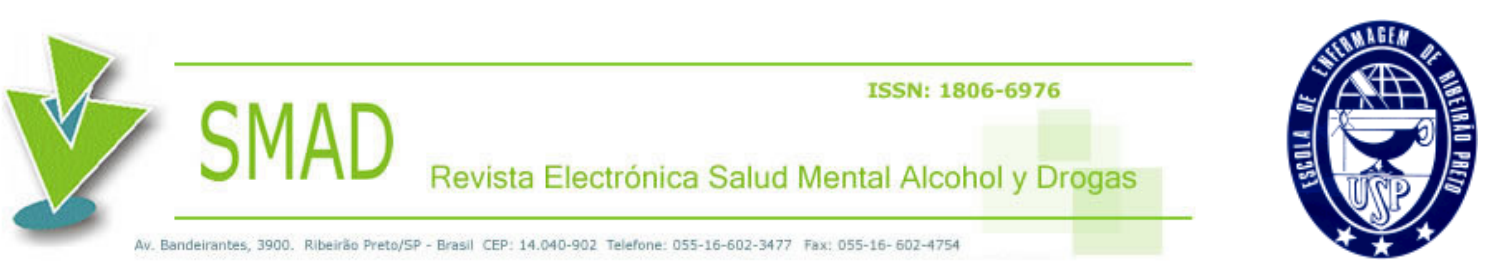

desconhecidas, e Fator 5 - autocontrole da agressividade a situações aversivas, reunindo itens de reação a estimulações aversivas do interlocutor, com controle da raiva e agressividade.

\section{Resultados e Discussão}

A média de idade, tanto do grupo de usuários de maconha como do grupo de não usuários, corresponde a 17,07 anos $(\mathrm{dp}=1,75)$. Quanto aos dados sociodemográficos do grupo de usuários de maconha, 33,3\% (n=5) dos adolescentes moram com os pais, 53,3\% $(n=8)$ moram com apenas um dos pais, $13,3 \%(n=2)$ moram com outros familiares. No grupo de não usuários, 84,6\% (n=11) moram com os pais, 15,4\% (n=2) mora com outros parentes. Referente ao estado civil, todos são solteiros.

No grupo de usuários de maconha, 86,7 (n=13) não trabalham, enquanto 60\% (n=9) do grupo de não usuários trabalham. Essa variável evidencia diferenças entre os grupos. Com relação à renda familiar, no grupo de usuários $46,2 \%(n=6)$ não possuem renda e 23,1\% (n=3) afirmam ter renda entre $\mathrm{R} \$ 500$ e 1000. Já no grupo de não usuários, 40\% possuem renda de $\mathrm{R} \$ 500$ a 1000.

Quanto à escolaridade, no grupo de usuários, 86,7\% (n=13) estão estudando e no grupo de não usuários $100 \%(\mathrm{n}=15)$ estudam. Dos usuários, $80 \%(\mathrm{n}=12)$ possuem ensino fundamental incompleto, 6,7\% $(n=1)$ ensino fundamental completo, 20\% (n=3) cursam

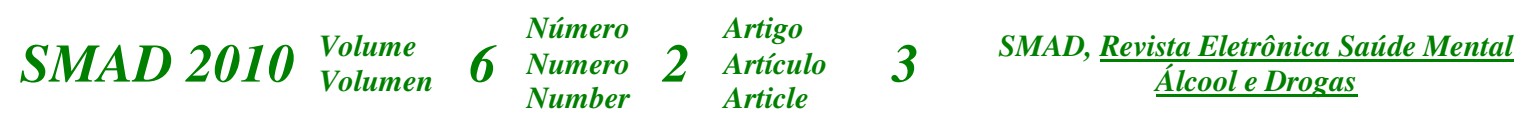



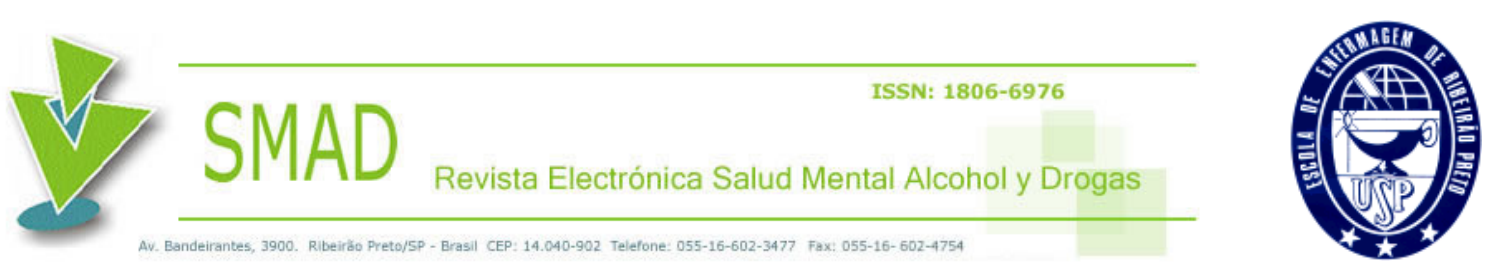

ensino médio e 6,7\% (n=1) ensino superior completo. Dos não usuários, $80 \% \quad(n=12)$ possuem ensino fundamental incompleto e $6,7 \%(n=1)$ cursam ensino médio.

Com relação a problemas na família, associados ao uso de drogas, 93,3\% (n=14) do grupo de usuários referem ter problemas familiares devido ao uso de substâncias, e no grupo de não usuários 46,7\% (n=7) têm o mesmo problema. A literatura corrobora esses resultados, apontando que um dos fatores que pode contribuir para o consumo de substâncias pelos adolescentes é o fato de familiares usarem drogas ${ }^{(5-6)}$.

Em relação à avaliação das habilidades sociais, os achados finais apontam que não houve diferenças estatísticas significativas no escore geral do IHS entre o grupo de usuários e não usuários, mas foram encontradas diferenças significativas em relação a um dos cinco fatores do IHS. O grupo de usuários de maconha apresentou desempenho mais prejudicado no Fator 5: autocontrole da agressividade a situações aversivas. Esse resultado aponta para a existência de maiores prejuízos nas habilidades em adolescentes usuários de maconha, evidenciando que esse grupo apresenta maior inabilidade para lidar com sentimentos e reações de agressividade, gerados em situações sociais que vivenciam. A Tabela 1 se refere à comparação entre as médias obtidas nos fatores do Inventário de Habilidades Sociais.

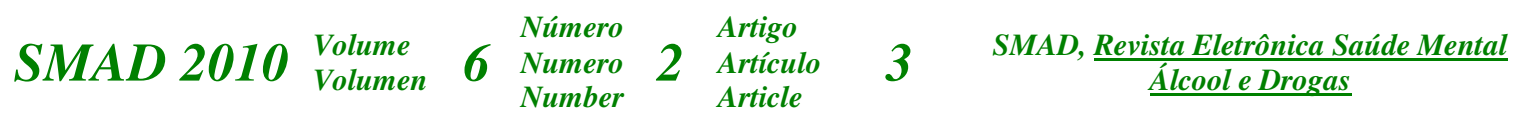



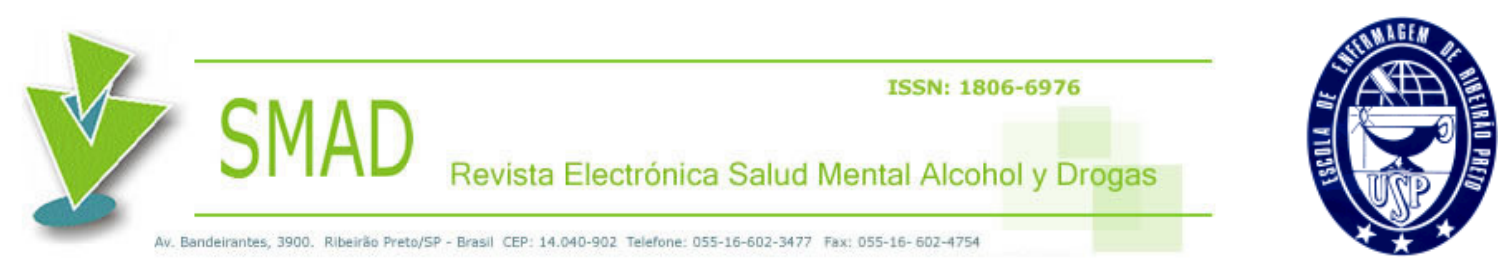

Tabela 1 - Comparação das médias e desvios padrão do escore total e dos fatores do IHS entre os grupos de usuários e não usuários de maconha $(n=30)$

\section{Usuários Não usuários}

\begin{tabular}{lccc} 
IHS & Posto médio & Posto médio & p valor \\
\hline Escore total & 11,67 & 17,77 &, 052 \\
Fator 1 & 13,43 & 15,73 &, 467 \\
Fator 2 & 14,03 & 15,04 &, 751 \\
Fator 3 & 12,43 & 16,88 &, 156 \\
Fator 4 & 12,20 & 17,15 &, 118 \\
Fator 5 & 10,87 & 18,69 &, $011 *$ \\
\hline \multicolumn{2}{c}{ p dado pelo Mann-Whitney } & $* \mathrm{p}<0,05$ significativo
\end{tabular}

No que se refere à avaliação da presença de sintomas depressivos, a média do escore total do grupo de usuários corresponde a 11,06 (dp: 10,01) e do grupo de não usuários a 6,15 (dp: 5,53), ou seja, o grupo de usuários apresenta maior intensidade de sintomas depressivos. Os resultados referentes às categorias estão na Tabela 2.

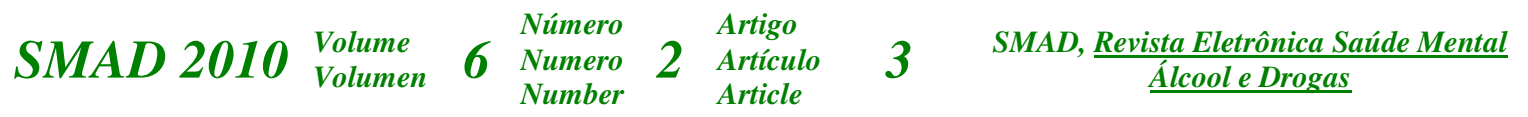


Tabela 2 - Distribuição percentual quanto à intensidade de sintomas depressivos

\section{Classificação}

\section{Usuários}

\section{Não usuários}

\begin{tabular}{lcccc} 
& $\mathbf{n}$ & $\mathbf{\%}$ & $\mathbf{n}$ & $\mathbf{\%}$ \\
\hline Mínima & 9 & 60 & 11 & 84,6 \\
Leve & 3 & 20 & 2 & 15,4 \\
Moderada & 8 & 17,8 & - & - \\
Grave & 1 & 6,7 & - & - \\
Total & 15 & 100 & 15 & 100 \\
\hline
\end{tabular}

Com relação aos sintomas de ansiedade, a média do escore total do grupo de usuários corresponde a 12,33 (dp: 9,37) e do grupo de não usuários a 5,30 (dp: 4,58). O escore geral foi maior no grupo de usuários de maconha, indicando maior intensidade de sintomas de ansiedade. Os resultados referentes à classificação estão na Tabela 3.

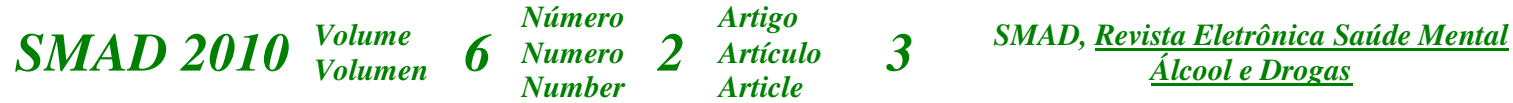


Tabela 3 - Distribuição percentual quanto à intensidade de sintomas de ansiedade

\begin{tabular}{lcccc} 
& \multicolumn{2}{c}{ Usuários } & \multicolumn{2}{c}{ Não usuários } \\
\cline { 2 - 5 } Classificação & n & $\%$ & $\mathbf{n}$ & $\%$ \\
\hline Mínima & 24 & 54,5 & 31 & 68,9 \\
Leve & 12 & 27,3 & 12 & 26,7 \\
Moderada & 7 & 15,9 & 1 & 2,2 \\
Grave & 1 & 2,2 & 1 & 2,2 \\
Total & 45 & 100 & 45 & 100 \\
\hline
\end{tabular}

Diversas pesquisas têm demonstrado ser bastante comum que adolescentes abusadores ou dependentes de drogas apresentem comorbidades. Ou seja, apresentem o transtorno por uso de substância psicoativa combinado com outro transtorno, tais como ansiedade e depressão ${ }^{(22)}$.

De acordo com a Tabela 4, é possível constatar diferença quanto ao desempenho dos participantes dos dois grupos na aplicação da Wechsler Intelligence Scale for Children - Third Edition (WISC-III) e da Wechsler Adult Intelligence Scale - Third Edition (WAISIII). Nos subtestes Código e Cubos, a média de desempenho no grupo de usuários foi mais baixa do que no grupo de não usuários, o que pode indicar prejuízos cognitivos, ainda não muito acentuados, devido ao pouco tempo de uso da substância psicoativa. Em estudo

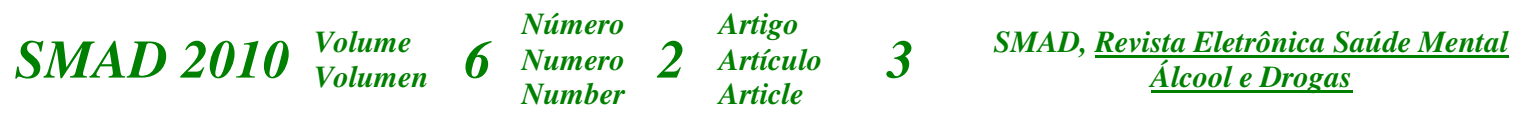


realizado com adolescentes usuários de maconha, foram observados que prejuízos nas capacidades cognitivas podem não ser percebidos em curto prazo, devido ao pouco tempo de uso da substância, já que os déficits se acentuam a longo prazo ${ }^{(23)}$.

Tabela 4 - Média e desvio padrão dos substestes das Escalas Weschler (screening cognitivo)

\begin{tabular}{lccccc}
\hline Subtestes & $\begin{array}{c}\text { Usuários } \\
\text { Média }\end{array}$ & $\mathbf{d p}$ & $\begin{array}{c}\text { Não usuários } \\
\text { Média }\end{array}$ & $\mathbf{d p}$ & $\mathbf{p}$ \\
\hline Vocabulário & 8,73 & 3,51 & 8,53 & 2,20 &, 85 \\
Códigos & 7,54 & 4,03 & 8,80 & 2,73 &, 35 \\
Dígitos & 8,57 & 3,95 & 8,93 & 2,03 &, 78 \\
Cubos & 9,93 & 3,01 & 11,20 & 2,70 &, 23 \\
\hline p dado pelo teste t de Student & & & &
\end{tabular}

\section{Considerações Finais}

O presente estudo indica a existência de prejuízos maiores no grupo de usuários de maconha nas habilidades sociais, bem como nos aspectos cognitivos, além da presença de mais sintomas indicativos de ansiedade e depressão, quando comparados ao grupo de não usuários.

Em relação às habilidades sociais, conclui-se, por meio desses resultados, que a área

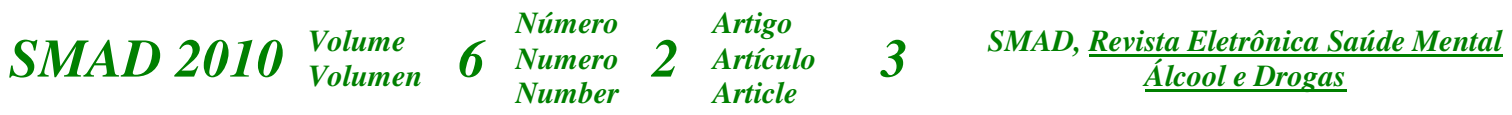



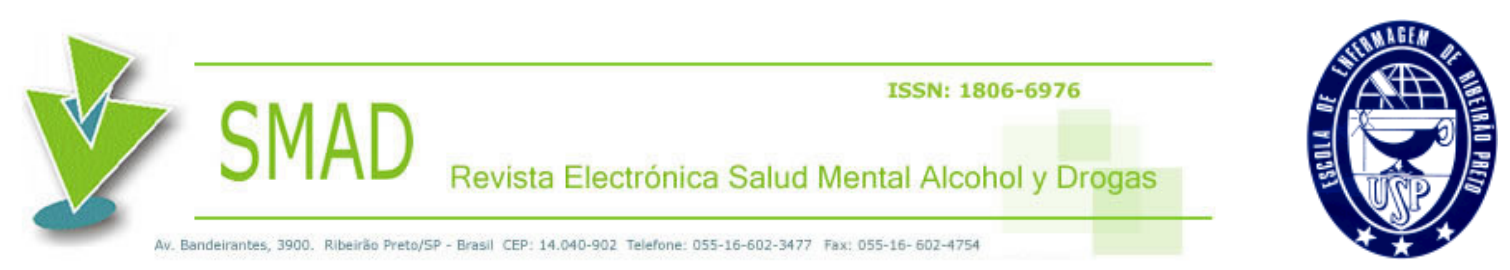

mais deficitária na população de usuários de maconha relaciona-se ao autocontrole da agressividade a situações aversivas, denotando que adolescentes que fazem uso de substâncias psicoativas tendem a apresentar maior inabilidade para lidar com sentimentos e reações gerados nas situações sociais, o que pode contribuir para a busca da substância como comportamento não assertivo de enfrentamento de tais dificuldades. Outros estudos relatam resultados semelhantes em amostra de 98 adolescentes, na qual também foram constatados maiores prejuízos no grupo de usuários de substâncias no screening cognitivo e na presença de sintomas de ansiedade e depressão, surgindo diferenças estatísticas significativas. Porém, houve desempenho mais prejudicado em dois fatores do IHS, Fator 4 - autoexposição a desconhecidos ou a situações novas e Fator 5- autocontrole da agressividade em situações aversivas, o que também evidenciou que adolescentes usuários de substância apresentam mais prejuízos nas habilidades do que adolescentes não usuários de substâncias ${ }^{(24)}$.

Os achados apontam que o uso de substâncias pelos familiares pode aumentar as probabilidades de o jovem também consumir drogas, o que foi evidenciado com pontuação do grupo de usuários bem mais elevada nesse aspecto. Esses resultados corroboram estudos realizados, sugerindo que o consumo de drogas pelos pais pode ser fator de risco para problemas com as drogas, em adolescentes. O processo de aprendizagem social negativa ocorre, pois a criança cresce observando adultos lidando com seus próprios problemas através do uso de drogas e aprenderá esse comportamento como única habilidade de enfrentamento $^{(25)}$.

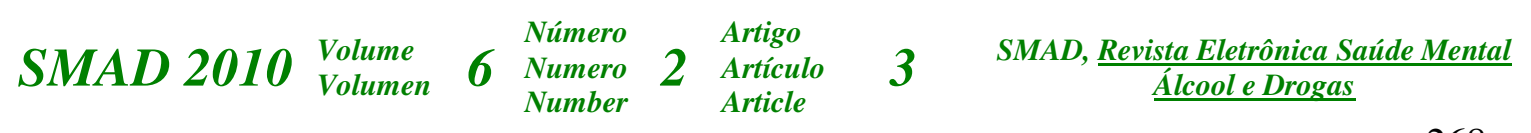



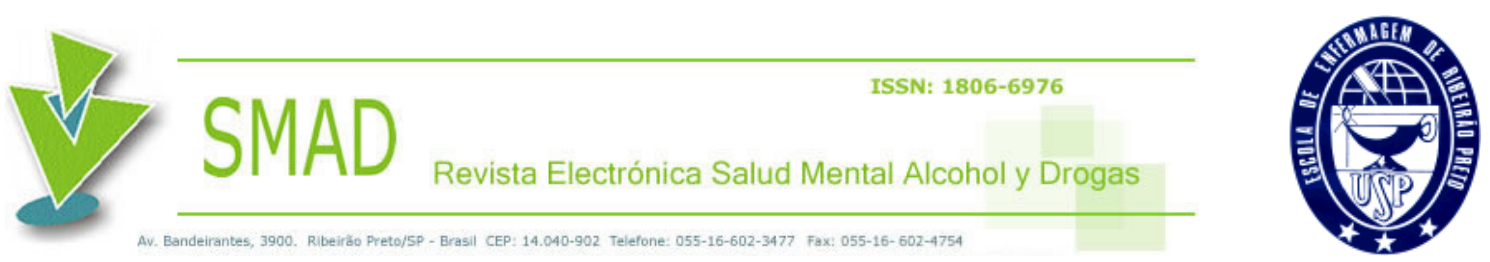

Os dados acima estão de acordo com estudo desenvolvido com alcoolistas, no qual foi identificada forte associação entre hábitos de bebida do pai e padrão de bebida do sujeito, reforçando o pressuposto de que o consumo pelos pais pode ser fator de risco para o uso de substâncias ${ }^{(26)}$. Esses dados vêm ao encontro da teoria da aprendizagem social, a qual considera que os comportamentos aditivos podem ser considerados como hábitos hiperaprendidos que podem ser analisados e modificados da mesma maneira que os demais hábitos ${ }^{(27)}$.

A partir dos dados obtidos, pode-se observar que a experimentação e o uso regular de substâncias psicoativas, como a maconha, vêm aumentando. Conhecer o seu alcance e os padrões de uso nos diversos segmentos da população representa recurso indispensável para delinear indicadores e para a escolha de medidas de intervenção adequadas e eficazes. Dessa forma, intervenções voltadas ao treinamento de habilidades sociais poderiam estimular o desenvolvimento de habilidades de enfrentamento dos jovens, voltadas às pressões sociais de seus pares.

Nesse sentido, o presente trabalho sugere a necessidade de novos estudos voltados à intervenção na área de habilidades sociais, em adolescentes usuários de drogas psicoativas, visto que se constata que o uso de substância está ocorrendo cada vez mais em idade precoce e com aumento preocupante em todo o mundo. Sendo também importante a avaliação das funções cognitivas nessa população, para que se possa focar em intervenções direcionadas para as áreas mais afetadas.

Os resultados demonstraram a relevância da identificação de possíveis

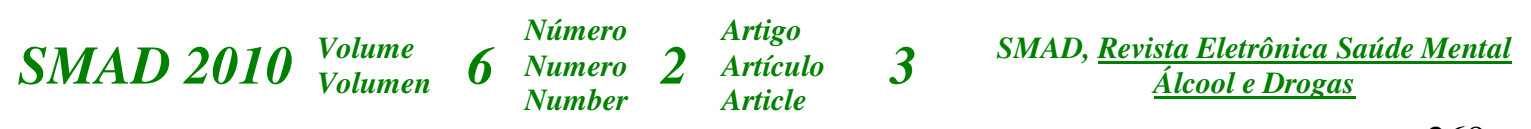



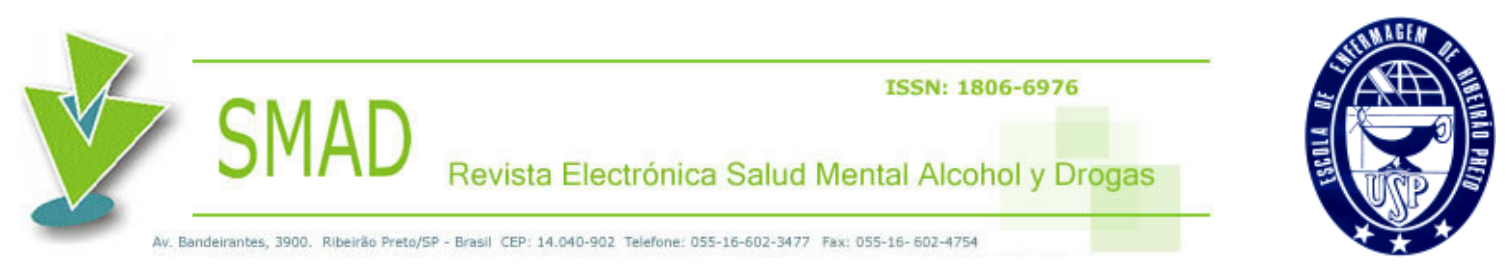

comorbidades relacionadas ao uso de substâncias, bem como dos fatores que contribuem para que os adolescentes iniciem o abuso de substâncias, vislumbrando opções adequadas de tratamento a essa população. Trabalhos preventivos de redução da motivação para o uso de substâncias, além do treinamento das habilidades sociais de crianças e adolescentes, podem representar fatores de proteção ao consumo de substâncias e futuro desenvolvimento de transtornos psicológicos.

\section{Referências}

1. Bolognini M, Plancherel B, Laget J, Halfon O. Adolescent's suicide attempts: Populations at risk, vulnerability, and substance use. Substance Use \& Misuse. 2004; 38: 1651-69.

2. Kaminer Y, Szobot C. O tratamento de adolescentes com transtornos por uso de substâncias psicoativas. In: Pinsky I, Bessa M, editores. Adolescência e Drogas. São Paulo: Contexto; 2004. p. 164-78.

3. Carlini EA, Galduróz JC, Noto AR, Carlini CM, Oliveira LG, Nappo SA, et al. II Levantamento Domiciliar sobre o Uso de Drogas Psicotrópicas no Brasil: um estudo envolvendo as 108 maiores cidades do país, 2005. São Paulo: CEBRID/UNIFESP; 2007.

4. American Psychiatric Association, APA. Manual Diagnóstico e Estatístico de Transtornos Mentais DSM-IV-TR. 4ª ed. rev. Porto Alegre: Artmed; 2002.

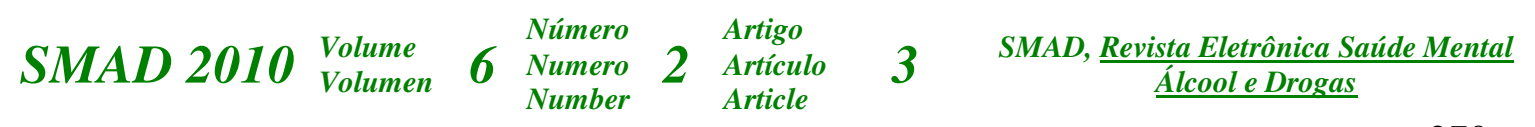


5. Aliane PP, Lourenço LM, Ronzani TM. Estudo comparativo das habilidades sociais de dependentes e não dependentes de álcool. Psicol Estudo. 2006;11(1):83-8.

6. Schroeder JL, Johnson GE. Accessing Substance Abuse Prevention Programs for Schools. Intervent School Clin. 2009;44(4):234-40.

7. Caballo VE. Manual de avaliação e treinamento das habilidades sociais. São Paulo: Livraria Santos Editora; 2003.

8. Del Prette ZAP, Del Prette A. Transtornos psicológicos e habilidades sociais. In: Guillardi HJ, organizador. Sobre Comportamento e Cognição: Contribuições da construção da teoria do comportamento. São Paulo: ESETec; 2002. p. 377-86.

9. Lucca E. Habilidade Social: uma questão de qualidade de vida. In: Psicologia.com.pt: O Portal dos Psicólogos [On-line] 2004. [acesso em: 06 ago 2009]. Disponível: http://www.psicologia.com.pt/artigos/textos/A0224.pdf .

10. Falcone EMO. Contribuições para o treinamento de habilidades de interação. In: Guilhardi HJ, Madi MBBP, Queiroz PP, Scoz MC, organizadores. Sobre comportamento e cognição:contribuições para a construção da teoria do comportamento. Santo André: ESETec; 2002. p. 91-104.

11. Mestre M. Habilidades Sociais e Comunicação. Psicol Argumento. 2001;19(29):33-7.

12. Oliveira MS. Abordagens psicoterápicas. In: Pulcherio G, Bicca C, Silva FA, organizadores. Álcool, outras drogas, informação: o que cada profissional precisa saber. São Paulo: Casa do Psicólogo; 2002. p. 125-45.

13. Del Prette ZAP, Del Prette A, Barreto MCM, Bandeira M, Rios-Saldaña MR, Ulian SMAD $2010 \begin{aligned} & \begin{array}{l}\text { Volume } \\ \text { Volumen }\end{array} \\ & \text { Solumero }\end{aligned} \quad \begin{aligned} & \begin{array}{l}\text { Número } \\ \text { Numero } \\ \text { Number }\end{array} \\ & 2\end{aligned} \begin{aligned} & \begin{array}{l}\text { Artigo } \\ \text { Artículo } \\ \text { Article }\end{array} \\ & \text { SMAD, Revista Eletrônica Saúde Mental }\end{aligned}$ 

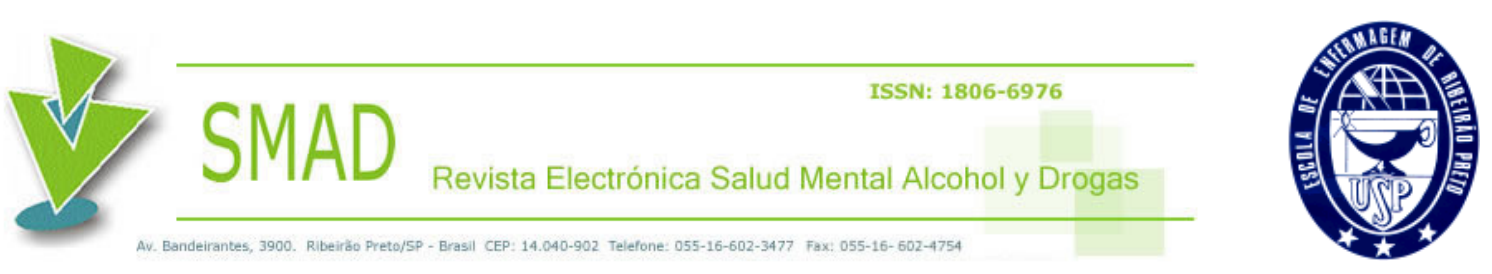

ALAO, et al. Habilidades sociais de estudantes de Psicologia: Um estudo multicêntrico. Psicol Reflex Crít. 2004;17(3):341-50.

14. Inderbitzen-Nolan HM, Anderson ER, Johnson HS. Subjective versus objective behavioral ratings following two analogue tasks: A comparison of socially phobic and nonanxious adolescents. J Anxiety Dis. 2007;21:76-90.

15. Alexandre, NL, Del Rio, MP, Pol AP. Estrategias de afrontamiento: factores de protección en el consumo de alcohol, tabaco e cannabis. Adicciones. 2004;16(4):1-6.

16. Stephens PC, Sloboda Z, Stephens RC, Teasdale B, Grey SF, Hawthorne RD, et al. Universal school-based substance abuse prevention programs: Modeling targeted mediators and outcomes for adolescent cigarette, alcohol and marijuana use. Drug Alcohol Depend. 2009;102:19-29.

17. Oliveira MS, Werlang BSG, Wagner MF. Expectativas acerca del alcohol y su relación con el patrón de consumo de bebida. Rev Arg Clín Psicol. 2006;15(2):99-107.

18. Victoir A, Eertmans A, Van den Bergh O, Van den Broucke S. Association of substance-use behaviours and their social-cognitive determinants in secondary school students. Health Educ Res. 2007;22(1):81-94.

19. Del Prette A, Del Prette ZAP. Inventário de Habilidades Sociais: manual de aplicação, apuração e interpretação. São Paulo: Casa do Psicólogo; 2001.

20. Cunha JA. Psicodiagnóstico - 5. Porto Alegre: Artes Médicas; 2000.

21. Cunha JA. Manual da versão em português das escalas Beck. São Paulo: Casa do Psicólogo; 2001.

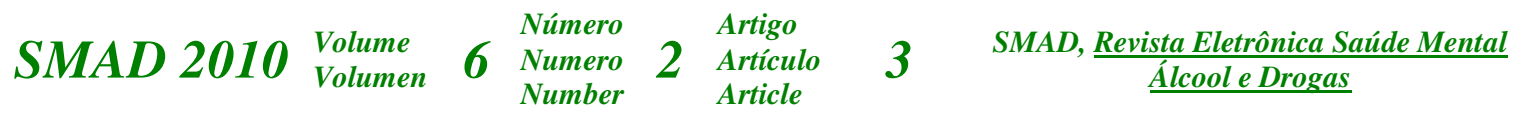


22. Bessa MA. Quando o uso de drogas ocorre com outros transtornos psiquiátricos. In: Pinsky I, Bessa MA, editores. Adolescência e drogas. São Paulo: Contexto; 2004. p. 12450.

23. Rigoni MS, Oliveira MS, Moraes JF, Zambon L F. O consumo de maconha na adolescência e as funções cognitivas. Psicol Estudo. 2007;12:267-75.

24. Wagner MF, Oliveira MS. Estudo das habilidades sociais em adolescentes usuários de maconha. Psicol Estudo. 2009;14(1):101-10.

25. Silva VA, Aguiar AS, Felix F, Rebello GP, Andrade RC, Mattos HF, et al. Estudo brasileiro sobre abuso de substâncias por adolescentes: fatores associados e adesão ao tratamento. Rev Bras Psiquiatr. 2003;25(3):133-8.

26. Oliveira MS, Werlang BSG, Wagner MF. Relação entre o consumo de álcool e hábitos paternos de ingestão alcoólica. Bol Psicol. 2007;62(127):205-14.

27. Marlatt GA. Prevenção de Recaída: racionalidade teórica e visão geral do modelo. In: Marlatt GA, Gordon JR. Prevenção da Recaída: estratégia e manutenção no tratamento de comportamentos adictivos. São Paulo: Artes Médicas; 1993. p. 3-63.

Recebido em: 24/09/2009

Aprovado em: 15/03/2010

\section{Como citar este artigo:}

Wagner MF, Silva JG, Zanettelo LB, Oliveira MS. O uso da maconha associado ao déficit de habilidades sociais em adolescentes. SMAD, Rev. Eletrônica Saúde Mental Álcool Drog. (Ed. port.) [Internet]. 2010 [acesso em: dia mês abreviado com ponto ano] ; 6(2):255-73. Disponível em: Endereço Eletrônico Visitado.

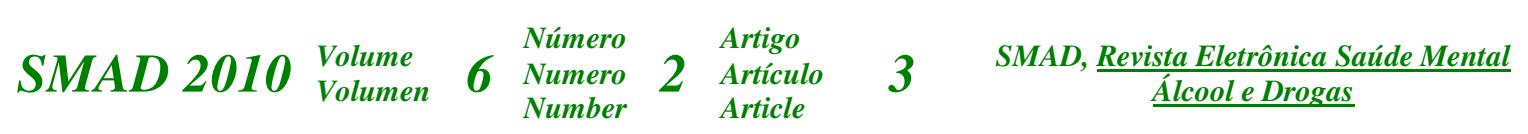

